\title{
Exon deletions of the EP300 and CREBBP genes in two children with Rubinstein-Taybi syndrome detected by aCGH
}

\author{
Anne Chun-Hui Tsai ${ }^{1}$, Cherilyn J Dossett ${ }^{1}$, Carol S Walton ${ }^{1}$, Andrea E Cramer ${ }^{2}$, Patti A Eng ${ }^{3}$, \\ Beata A Nowakowska ${ }^{3,4}$, Amber N Pursley ${ }^{3}$, Pawel Stankiewicz ${ }^{3,4}$, Joanna Wiszniewska ${ }^{3}$ and Sau Wai Cheung ${ }^{\star, 3}$ \\ We demonstrate the utility of an exon coverage microarray platform in detecting intragenic deletions: one in exons 24-27 of \\ the EP300 gene and another in exons 27 and 28 of the CREBBP gene in two patients with Rubinstein-Taybi syndrome (RSTS). \\ RSTS is a heterogeneous disorder in which $\sim 45-55 \%$ of cases result from deletion or mutations in the CREBBP gene and an \\ unknown portion of cases result from gene changes in EP300. The first case is a 3-year-old female with an exonic deletion of \\ the EP300 gene who has classic facial features of RSTS without the thumb and great toe anomalies, consistent with the milder \\ skeletal phenotype that has been described in other RSTS cases with EP300 mutations. In addition, the mother of this patient \\ also had preeclampsia during pregnancy, which has been infrequently reported. The second case is a newborn male who has \\ the classical features of RSTS. Our results illustrate that exon-targeted array comparative genomic hybridization (aCGH) is \\ a powerful tool for detecting clinically significant intragenic rearrangements that would be otherwise missed by aCGH platforms \\ lacking sufficient exonic coverage or sequencing of the gene of interest.
}

European Journal of Human Genetics (2011) 19,43-49; doi:10.1038/ejhg.2010.121; published online 18 August 2010

Keywords: EP300; intragenic deletion; intragenic dosage abnormalities; microarray; Rubinstein-Taybi

\section{INTRODUCTION}

Rubinstein-Taybi syndrome (RSTS) is characterized by growth retardation, dysmorphic features, skeletal abnormalities and mental retardation. ${ }^{1}$ Broad and angulated thumbs and halluces are considered hallmarks in the clinical diagnosis. Mutations in two genes, CREBBP and $E P 300$, have been identified in affected individuals. ${ }^{2,3}$ Individuals reported with mutations in EP300 have a milder skeletal phenotype, lacking typical broadening and angulation of the thumb and hallux. ${ }^{4}$ CREBBP and EP300 are ubiquitously expressed homologous proteins that act as transcriptional co-activators. Both genes are highly conserved and their proteins are thought to have two functions: formation of a bridge or scaffold between the DNA-binding transcription factors and the RNA polymerase II complex, and serving as histone acetyltransferases that open up the chromatin structure, a process that is essential for gene expression. ${ }^{5}$

Clinical implementation of array comparative genomic hybridization (aCGH) enables high-resolution human genome analyses, and the discovery of extensive copy-number variation $(\mathrm{CNV})$ has led to the identification of new microdeletion and microduplication syndromes also known as genomic disorders. ${ }^{6,7}$ With genome-wide studies identifying an increasing number of disease genes, such as in patients with autism spectrum disorders, ${ }^{8}$ and as copy-number changes in the genome can be observed as small as a single exon, ${ }^{9,10}$ this array has evolved to a wholegenome array that can simultaneously evaluate over 1700 genes with a resolution significant enough to detect intragenic changes.
Here we report two such cases of exonic deletions detected by this array platform; one in a male with RSTS resulting from an exon deletion within the CREBBP gene and the other in a female with RSTS resulting from a deletion within the EP300 gene. Although six other cases of EP300 RSTS have been previously reported, ${ }^{3,4,11,12}$ this is the first patient ascertained by microarray analysis in a clinical setting.

\section{CLINICAL REPORT}

Case 1

The child was first seen in the genetics clinic at the age of 34 months because of microcephaly, short stature, developmental delay and behavioral problems. She was born to a then G 1 P 0-1 22-year-old mother via $\mathrm{C}$-section at $\sim 33$ weeks. Prenatal exposures included maternal usage of Vicodin for migraines, Reglan for nausea, and multiple blood pressure medications for pregnancy-induced hypertension beginning at 14 weeks. Pregnancy was complicated by IUGR, thin placenta and premature labor at 28 weeks due to preeclampsia. Patient stayed in the NICU for 6 weeks for poor weight gain, which was partially attributed to a milk allergy. Overall, she was healthy with mild developmental delay and aggressive behaviors. She walked at 18 months and only has a few words and signs at 34 months of age. EEG and MRI were normal.

Physical examination noted a short, microcephalic girl who was hyperactive. Height was $83.9 \mathrm{~cm}(0.49 \%)$, weight was $11.2 \mathrm{~kg}(3 \%)$ and OFC was $44.5 \mathrm{~cm}(<2 \%)$. Dysmorphic facial features include hypotelorism, deep-set eyes with luxuriant eyelashes, downslanting

\footnotetext{
${ }^{1}$ The Children's Hospital, Section of Clinical Genetics and Metabolism, Denver, UC Denver, Aurora, CO, USA; ${ }^{2} 1600$ Republic Pkwy, Mesquite, TX, USA; ${ }^{3}$ Medical Genetics Laboratories, Department of Molecular and Human Genetics, Baylor College of Medicine, Houston, TX, USA; ${ }^{4}$ Department of Medical Genetics, Institute of Mother and Child, Warsaw, Poland

*Correspondence: Dr SW Cheung, Medical Genetics Laboratories, Department of Molecular and Human Genetics, Baylor College of Medicine, Houston, TX, USA. Tel: +1 713798 4991; Fax: +1 713798 4998; E-mail: scheung@bcm.edu
}

Received 8 March 2010; revised 13 May 2010; accepted 4 June 2010; published online 18 August 2010 
palpebral fissures, mild synophrys, hirsutism, prominent nasal tip, cupid-bow-shaped (beaked) upper lip and high-arched palate (Figure 1a). The arms, legs, fingers and toes were normal, except flat feet, hyperextensible joints and hypotonia. She demonstrated some repetitive and self-stimulating behaviors.

\section{Case 2}

This child was ascertained at birth with obvious stigmata of RSTS. He was a full-term male infant, delivered vaginally to a G3 P1 A1 34-year-old female after an unremarkable gestation. Maternal history was significant for rheumatoid arthritis for which she received methotrexate before conception. Family pedigree was unremarkable and consanguinity was denied. Birth growth parameters were normal. The NICU transfer was initiated upon development of respiratory distress and cardiac murmur, subsequently identified as due to patent ductus arteriosus and patent foramen ovale. Facial features are compatible with RSTS as shown in Figure 1b. Undescended unilateral testis, hypoplastic scrota and penis were identified, the latter responding well to testosterone injection. Microcephaly developed postnatally.

\section{MATERIALS AND METHODS}

\section{Patients}

Chromosomal microarray analysis Version 8 OLIGO (CMA V8 OLIGO) with exon coverage was performed on $\sim 3000$ samples that were referred to the Kleberg Cytogenetic Laboratory at Baylor College of Medicine (BCM) during the first 6 months of the clinical application of this array platform (June to December 2009). Informed consent approved by the Institutional Review Board for Human Subject Research at BCM was obtained from their families.

\section{DNA samples}

DNA was extracted from whole blood using the Puregene DNA extraction kit (Gentra, Minneapolis, MN, USA) according to the manufacturer's instructions.

\section{Clinical CMA}

The CMA V8 OLIGO is a custom designed array with $\sim 180000$ oligonucleotides of $60 \mathrm{bp}$ manufactured by Agilent Technologies Inc. (Santa Clara, CA, USA). This array selects the best performing oligos from the electronic library from Agilent and probes for virtually all the known microdeletion or microduplication syndromes, as well as the pericentromeric and subtelomeric regions as previously described in version $7 .{ }^{13}$ In addition, more than 1700 genes throughout the genome have coverage for every exon. On average, there are 4.5 oligos per exon and intron coverage if the intron is $>10 \mathrm{~kb}$. However, probe coverage for an individual exon may be less if the exon is small or there are few oligos that meet the selection criteria available from the electronic library of Agilent. The entire genome (between disease regions) is covered with an average resolution of $30 \mathrm{~kb}$ excluding repetitive sequences as determined through a combination of bioinformatics and computation. Although the majority of genes selected for exonic coverage are known disease genes, candidate genes implicated in autism, epilepsy and heart defects are also included in the array. The procedures for DNA digestion, labeling and hybridization for the oligo arrays were performed according to the manufacturers' instructions with some modifications. ${ }^{14}$ The slides were scanned into image files using an Agilent G2565 laser scanner. Microarray image files of oligo arrays were quantified using Agilent Feature extraction software (v9.0), and text file outputs from the quantitation analysis were imported into our in-house analysis package for copy-number analysis, as previously described. ${ }^{14}$

\section{High-density research aCGH analysis of chromosome 22}

The chromosome 22 array was designed to cover the entire long arm of chromosome 22 by selecting oligos from the e-library supplied by Agilent. This array contains 43099 arrayed 60-mer oligonucleotides, representing a compiled view of the long arm of chromosome 22 at an average resolution of $1 \mathrm{~kb}$ while excluding the regions with segmental duplication. The procedures for DNA digestion, labeling and hybridization were performed according to the manufacturer's instructions with some modifications. ${ }^{14}$ This array was used to confirm the deletion of EP300 in patient 1. a
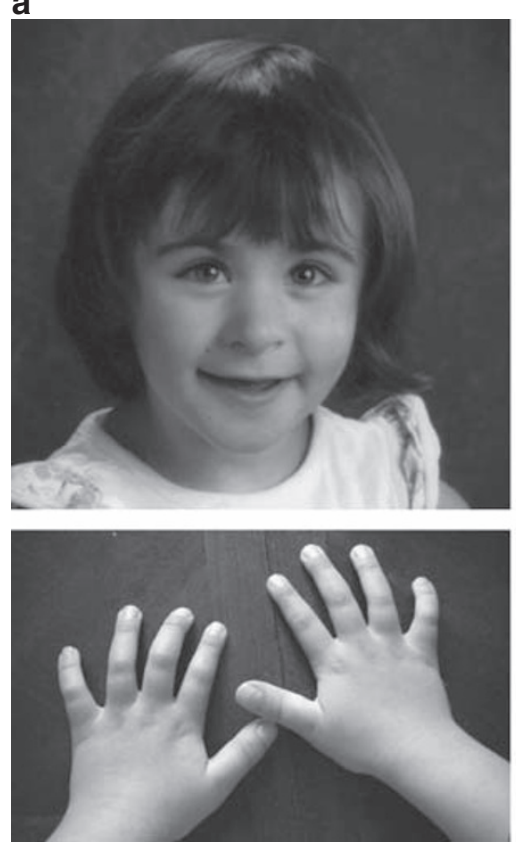

b

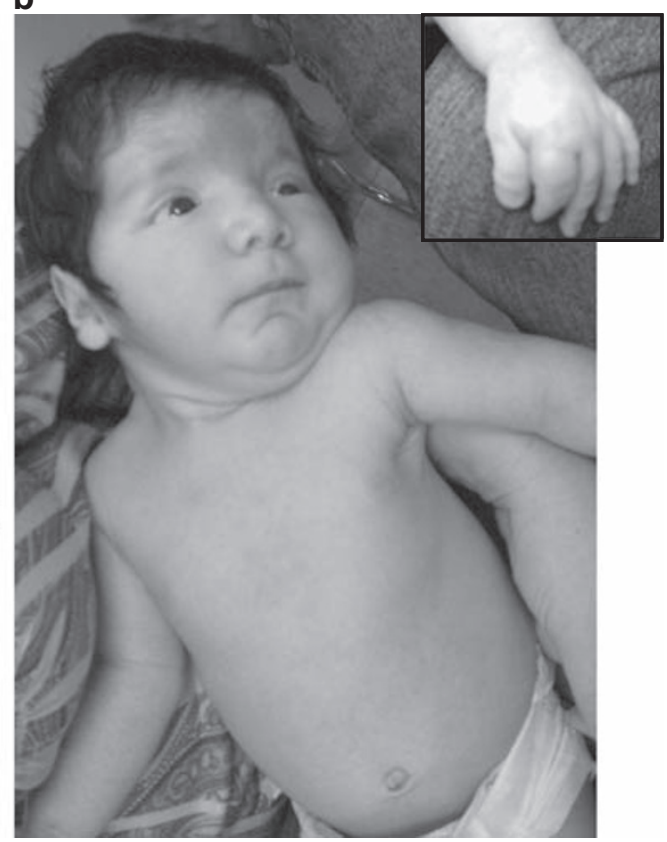

Figure 1 Clinical photos of the patients. (a) Case 1: Dysmorphic facial features include hypotelorism, deep-set eyes with luxuriant eyelashes, bushy eyebrows, synophrys, hirsutism, prominent nasal tip, cupid-bow-shaped/beaked upper lip and high-arched palate. (b) Case 2: Facial features of case 2 include hirsutism, low hairlines, glabellar nevus flammeus, ptosis, epicanthal folds, downslanting palpebral fissures, nasal septum below alae nasi and microstomia. Thumbs are broad and angulated; palmar creases are deep and simple. Halluces are broad but not angulated. Persistent fetal pads were noted on all fingers. 


\section{MLPA analysis}

Multiplex ligation-dependent probe amplification (MLPA) analysis was performed using commercially available SALSA MLPA kits, designed to detect deletions/ duplications in patients with RSTS (P333-A1 EP300 and P313-CREBBP, MRCHolland, Amsterdam, The Netherlands). ${ }^{15}$ Analysis was performed according to the manufacturer's protocol. The amplification products were separated by capillary electrophoresis using ABI 3730xl genetic analyzer (Applied Biosystems, Carlsbad, CA, USA). Data were visually inspected and analyzed using GeneMarker software v1.5 (SoftGenetics, LLC, State College, PA, USA).

\section{RESULTS}

Case 1

CMA V8 OLIGO revealed a loss in copy number of the long arm of chromosome 22 at sub-band 22q13.32 encompassing $\sim 5 \mathrm{~kb}$ within the EP300 gene and a gain in copy number on the long arm of chromosome 21 encompassing $\sim 160 \mathrm{~kb}$ (Figure 2a). Each of the oligos (red square) in the deleted region is displayed in the UCSC genome browser (build HG 18, http://genome.ucsc.edu/) corresponding to the four deleted exons of the EP300 gene (exons 24-27) (Figure 2b). The breakpoint for the deletion within the EP300 gene was further narrowed to be $4.43 \mathrm{~kb}$ using a high-resolution chromosome 22-specific array. The deletion breakpoint was mapped between 39893478 and $39894339 \mathrm{bp}$ and the distal breakpoint between 39896990 and 39897907 bp (data not shown). The deletion within the EP300 gene was also confirmed by MLPA to be from exons 24 to 27 (Figure 2c). The gain in copy number on the long arm of chromosome 21 (Figure 2a) was later found to be inherited from the phenotypically normal mother and therefore thought to be likely benign. Parental CMA studies showed the deletion involving EP300 is de novo. The parental MLPA studies using the same probes selected from the region encompassing the EP300 gene were also normal, consistent with the CMA findings.

\section{Case 2}

Routine chromosome analysis demonstrated a normal male chromosome complement: 46,XY. CMA revealed a loss in copy number in the 16 p13.3 region of the short arm of chromosome 16, spanning a minimum of $5 \mathrm{~kb}$ and a maximum of $6 \mathrm{~kb}$, harboring exons 27 and 28 of the CREBBP gene (Figures $3 \mathrm{a}$ and $\mathrm{b}$ ), which was verified by MLPA analysis (Figure 3c), consistent with the clinical indication of RSTS (OMIM 180849). In addition, CMA revealed a gain in copy number in the Xp22.31 region of the short arm of chromosome $\mathrm{X}$, spanning a minimum of $248 \mathrm{~kb}$ and a maximum of $358 \mathrm{~kb}$ (Figure 3a), which was confirmed by FISH analysis. Parental CMA studies were performed and it was confirmed that the deletion involving exons 27 and 28 is de novo, whereas the $\mathrm{CNV}$ on chromosome $\mathrm{X}$ was inherited from the phenotypically normal mother. This $\mathrm{CNV}$ on chromosome $\mathrm{X}$ has also been observed in the genomic variation database (http://projects. tcag.ca/variation/), indicating that it is also present in the normal population and therefore thought to be likely benign. In addition, parental MLPA studies for exons 27 and 28 of the CREBBP gene were also normal, consistent with the CMA findings.

\section{DISCUSSION}

With the introduction of a microarray platform with exon coverage, we demonstrate the utility of this technology in detecting intragenic deletions: one in exons 24-27 of the EP300 gene and another in exons 27 and 28 of the CREBBP gene. These results illustrate that exontargeted aCGH is a powerful tool for detecting clinically significant

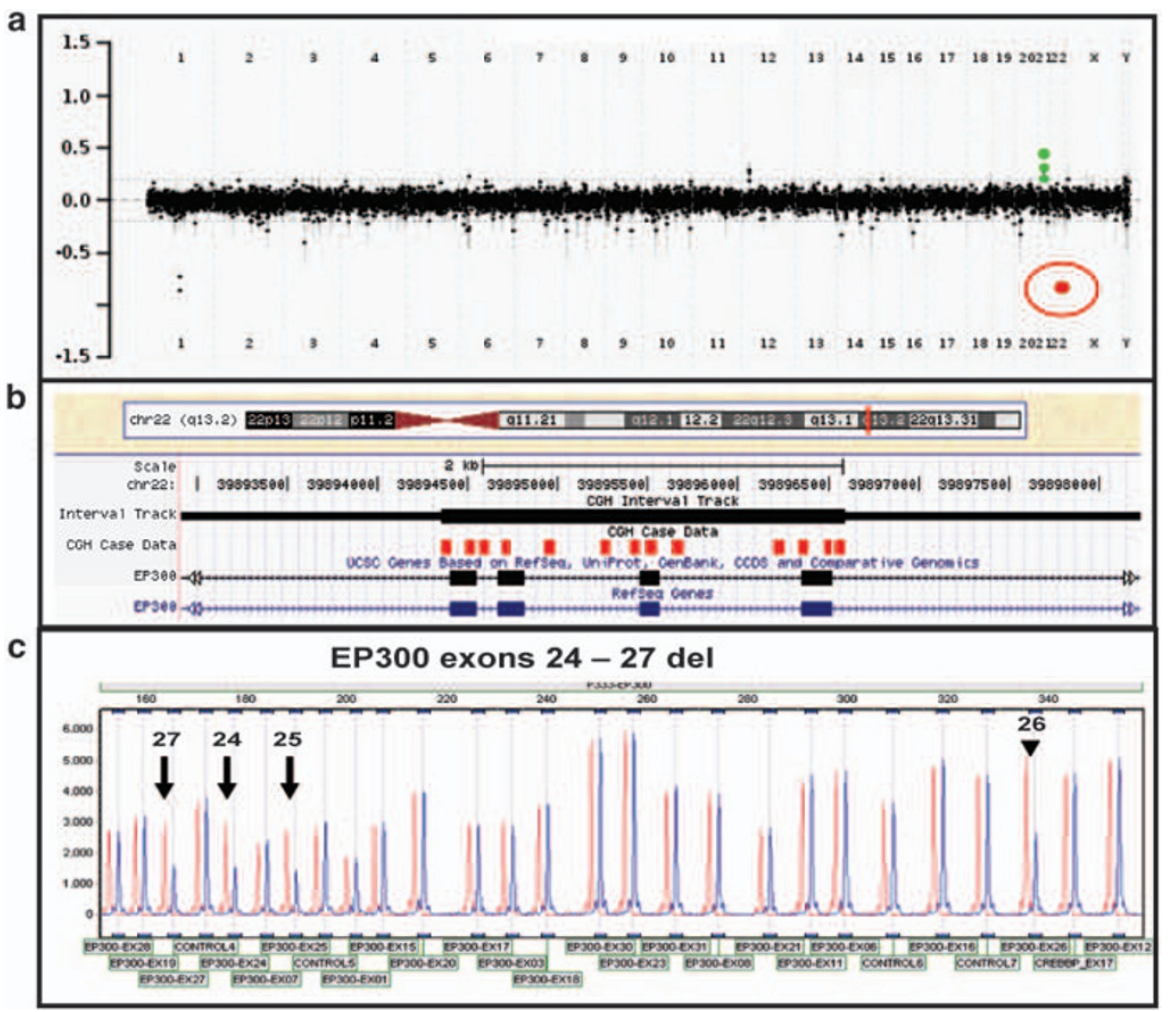

Figure 2 (a) Profile of the microarray analysis showing the deleted region as indicated in the red circle (the gain on chromosome 21 as shown in green dots is also present in the mother (data not shown)). (b) Each of the oligos (red square) in the deleted region is displayed in the UCSC genome browser (build HG 18) corresponding to the four deleted exons of the EP300 gene (exons 24-27). (c) The MLPA profile demonstrating the deletion of exons 24-27 of the EP300 gene as indicated by the arrow with the number for each exon. 


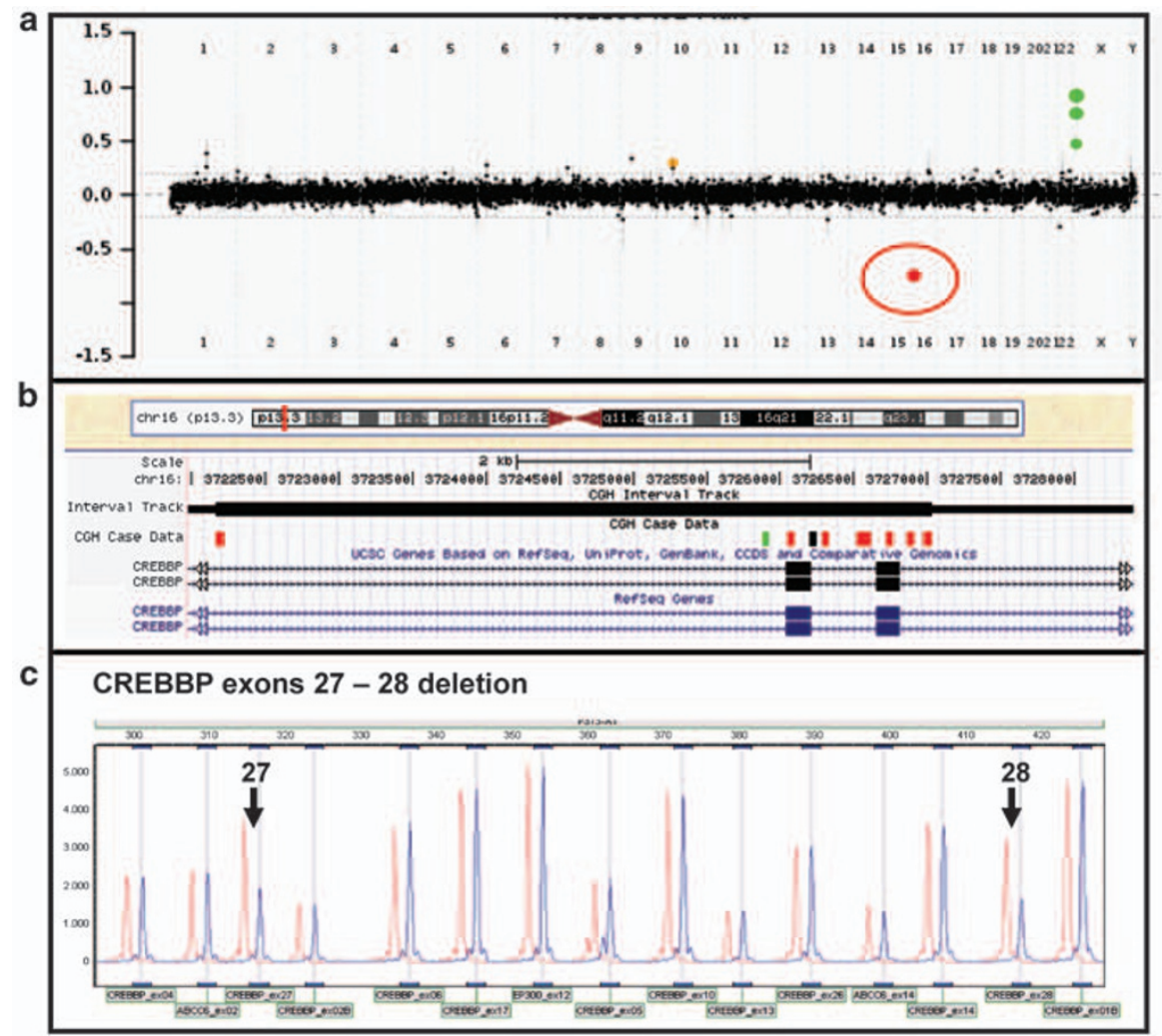

Figure 3 (a) Profile of the microarray analysis showing the deleted region as indicated in the red circle (the gain on Xp as shown in green dots is also present in the mother (data not shown), whereas the yellow dot on chromosome 10 represent a highly polymorphic region, which is also present in the Toronto database). (b) The deleted oligos displayed in the UCSC genome browser (build HG 18) corresponding to exons 27 and 28 of the CREBBP gene. (c) The MLPA profile demonstrating the two deleted exons of the CREBBP gene (exons 27-28) as indicated by the arrow with the number for each exon.

intragenic rearrangements that would be otherwise missed by aCGH platforms lacking sufficient exonic coverage or sequencing of the gene of interest. The resolution of the current clinical array used in the clinical laboratory setting is about $300-500 \mathrm{~kb}$, which is not sufficient to detect intragenic changes. This version 8 array was designed with exon coverage to detect deletions and duplications with a resolution between $1000 \mathrm{bp}$ and $300 \mathrm{~kb}$. The continuing improvement of cost-effective high-density arrays enables detection of copy-number changes that are $<1000 \mathrm{bp}$ in size in the clinical setting. In fact, the design of the high-density region-specific exon array for specific disease genes such as Duchenne/Becker muscular dystrophy demonstrated the ability to detect deletions and/or duplications involving a single exon. ${ }^{10}$ An increasing number of new genomic disorders are being described as a result of detection of small intragenic copynumber changes, such as the MEF2 $C^{16}$ and $N R X N 1^{17}$ genes, from high-density clinical arrays such as the one used in this report. Although other technologies, such as MLPA, can also detect intragenic deletions/duplications, it requires that the clinical phenotype be obvious enough to indicate testing for a specific gene. Whereas, an array with this level of resolution does not have this limitation enhancing its value for diagnosing conditions with variable expressivity or that lack hallmark clinical findings.

A total of 3000 samples were referred and evaluated using this version of the exon array during a period of 6 months. The indications for the study population varied from dysmorphic features, moderate developmental delay, mental retardation, multiple congenital anomalies, congenital heart disease to no indication provided. A total of 25 cases with exon deletions were identified and confirmed by PCR or
MLPA and/or sequencing. Among them, three cases were detected with RSTS, although only one case (case 2 ) had the indication to rule out RSTS. The third case was not reported here due to lack of consent. However, the indications for this case were moderate developmental delay, mental retardation and dysmorphic features. CMA revealed a copy-number loss at band 16p13.3 spanning $\sim 10 \mathrm{~kb}$. This loss deletes exons 11-16 of the CREBBP gene. This 3.5-year-old child has normal thumbs, but his great toes appear disproportionately large bilaterally as is seen in RSTS. He also has micrognathia, inverted nipples and prominent globes.

RSTS is a complex autosomal dominant disease. Point mutations and small deletions or insertions of the CREBBP and EP300 genes $^{3,18,19}$, as well as deletions and duplications $>1000 \mathrm{bp}$ in length to megabases, have been shown to lead to RSTS. ${ }^{3,20}$ Stef et al ${ }^{21}$ also showed that a deletion as small as $3.3 \mathrm{~kb}$ involving a few exons of the $C R E B B P$ gene can be detected using a set of seven BACs and 34 cosmids region-specific array covering $2 \mathrm{Mb}$ in the $16 \mathrm{p} 13.3$ region. It has been well established that deletions involving the CREBBP gene are the causative mutation in $\sim 10 \%$ of RSTS patient. Traditionally, FISH analysis has been employed to detect these deletions. However, the resolution of FISH analysis is not sufficient for detecting smaller intragenic deletions. With the increased resolution, this exon-targeted array now offers a new opportunity to diagnose patients with RSTS due to copy-number changes in these two genes associated with RSTS in a single assay. It may also prove particularly helpful in diagnosing patients with EP300 copy-number changes as these patients often do not present with the skeletal findings that are a hallmark feature of this syndrome. Therefore, the RSTS provides us an excellent example to 


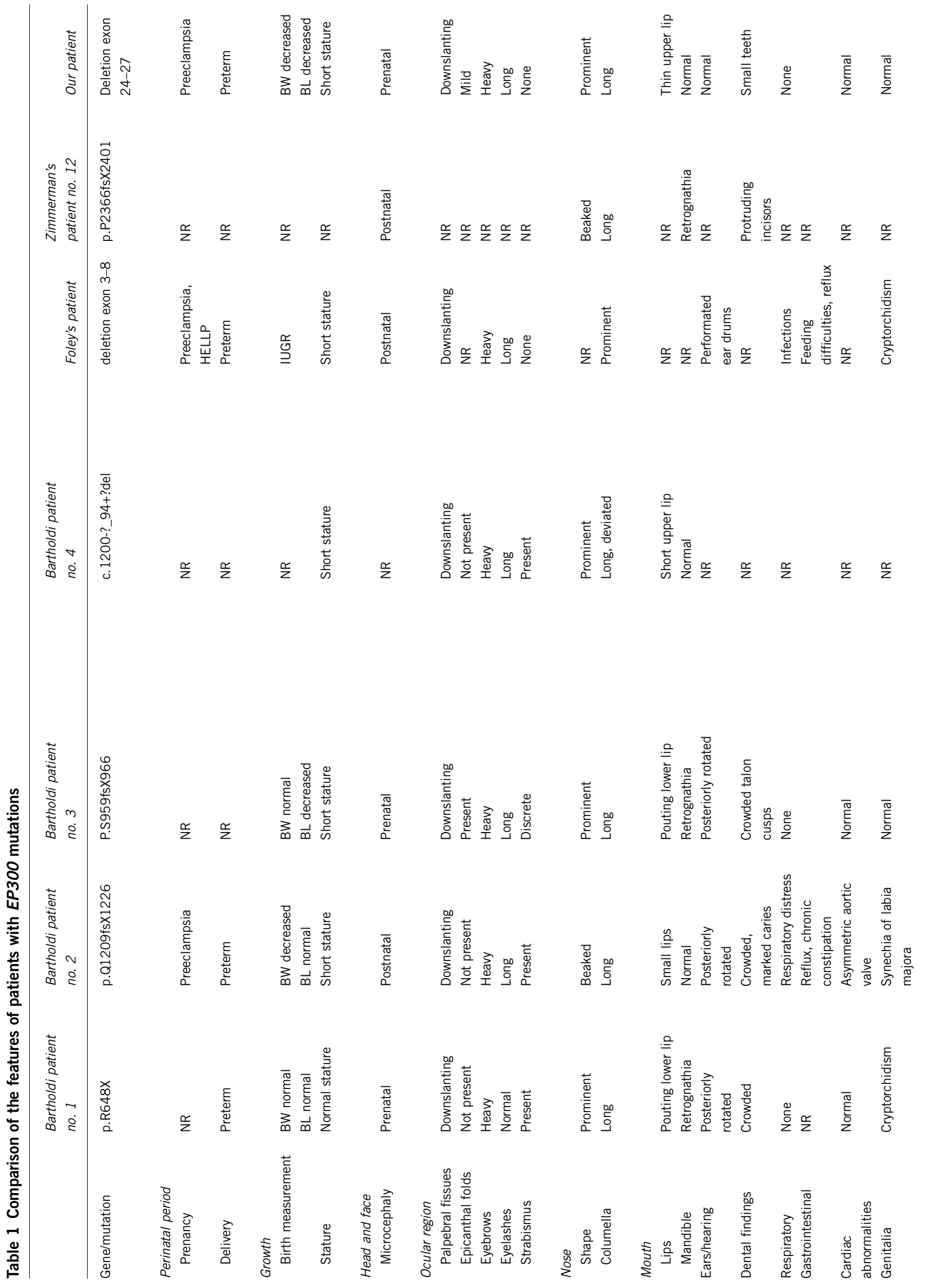




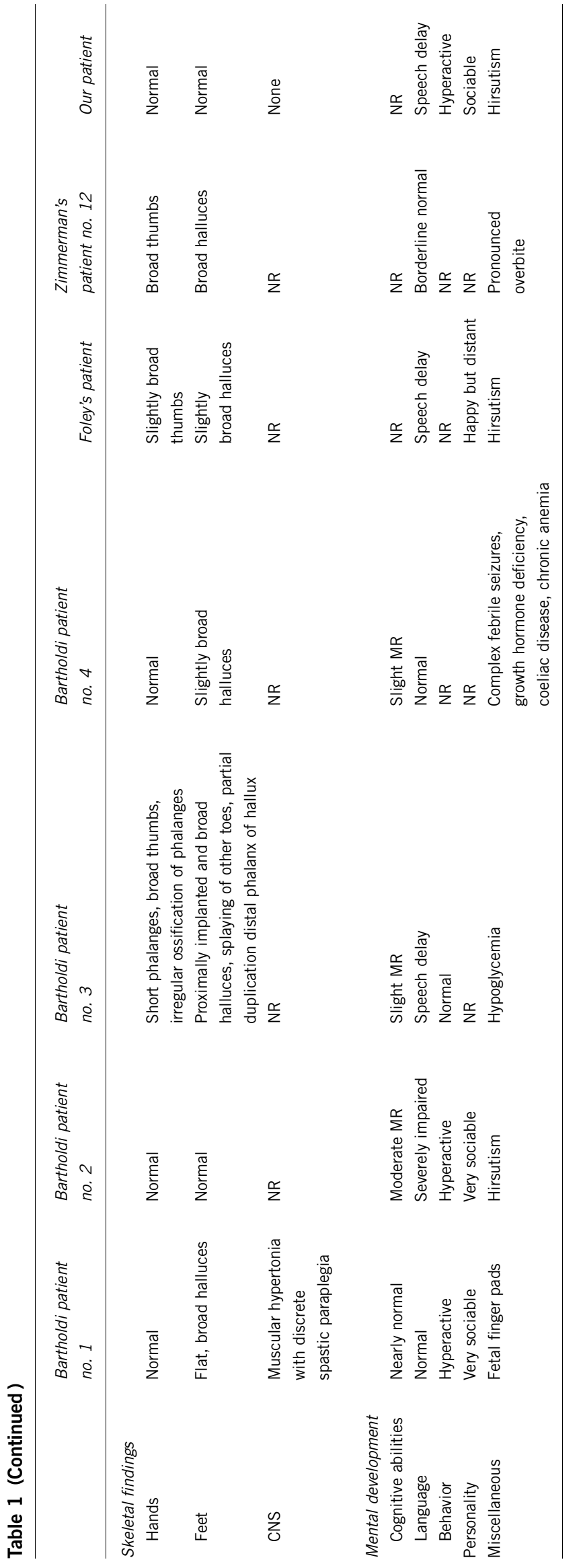

illustrate our capability in detecting small CNV to yet a higher level of resolution in the clinical setting, particularly if a specific gene cannot be targeted for testing based on the clinical findings.

Mutations and deletions in EP300 are rare causes of RSTS. Our case is only the seventh case reported to date. ${ }^{3,4,11,12}$ The true percentage of RSTS caused by EP300 alterations and the full phenotypic picture of EP300 RSTS is therefore unclear. The diagnosis in our case was considered because of the typical RSTS facial dysmorphism. However, due to the absence of broad and angulated thumbs and halluces, microarray testing was initiated first. CREBBP testing was to be performed as second-tier testing if the microarray was normal. The facial phenotype in our patient resembles the case (patient 2) described by Bartholdi et $a l^{4}$ and Foley et $a^{12}$ with very distinct arched bushy eyebrows and hirsutism over the lumbar region. Table 1 summarizes the reported cases of EP300 mutations, including pregnancy history and other physical and developmental features.

Interestingly, the mother of Bartholdi's second patient presented with preeclampsia, and the mother of Foley's patient had preeclampsia progressing to hemolysis, elevated liver enzymes and low platelets (HELLP) syndrome. ${ }^{4,12}$ Our patient's mother also developed preeclampsia; as she went into premature labor, it is possible that there was no chance for HELLP syndrome to develop. This was the third pregnancy of the seven reported cases to have preeclampsia, which we believe is a solid association. However, a literature search revealed no genes associated with EP300 that are known to also be associated with familial preeclampsia or HELLP syndrome. It is possible that as more genetic links are identified for preeclampsia and HELLP syndrome, an association with EP300 may become apparent. Of note, Lachmeijer et $a^{22}$ performed a genome scan using 293 polymorphic markers in 67 Dutch sib-pair families affected by preeclampsia, eclampsia or HELLP syndrome. Analysis in 38 preeclampsia families showed suggestive evidence for linkage on chromosome $22 \mathrm{q}$ at $32.4 \mathrm{~cm}$ (LOD score of 2.41), which is in close proximity to the EP300 gene and on chromosome $10 \mathrm{q}$ at $93.9 \mathrm{~cm}$ (LOD score of 2.38).

In conclusion, we report on two cases of RSTS due to exonic deletions detected by a clinical microarray analysis and only the seventh case of RSTS due to an EP300 mutation. Both our case and others in the literature with EP300 mutations have a modified RSTS phenotype, and, in particular, we confirm the milder skeletal phenotype. In addition, we propose that preeclampsia during pregnancy may be a new feature for RSTS caused by EP300 mutations. Together, these cases demonstrate the importance of considering EP300 RSTS in patients who have facial features of RSTS but lack broad and angulated thumbs and halluces. As this array can simultaneously evaluate for copy-number changes in both genes, it may be considered as the first line of testing for patients with suspected RSTS. This exon-targeted array may also replace the del/dup assay by MLPA to compliment sequencing analysis for these two genes. Our case demonstrates the utility of microarray for ascertaining atypical cases and further defining the phenotypic spectrum of RSTS.

\section{CONFLICT OF INTEREST}

The Department of Molecular and Human Genetics at Baylor College of Medicine derives revenue from the chromosomal microarray analysis offered in the Medical Genetics Laboratories.

1 Rubinstein JH, Taybi $\mathrm{H}$ : Broad thumbs and toes and facial abnormalities. A possible mental retardation syndrome. Am J Dis Child 1963; 105: 588-608. 
2 Petrij F, Giles RH, Dauwerse HG et al: Rubinstein-Taybi syndrome caused by mutations in the transcriptional co-activator CBP. Nature 1995; 376: 348-351.

3 Roelfsema JH, White SJ, Ariyurek Y et al: Genetic heterogeneity in Rubinstein-Taybi syndrome: mutations in both the CBP and EP300 genes cause disease. Am J Hum Genet 2005; 76: 572-580.

4 Bartholdi D, Roelfsema JH, Papadia F et al: Genetic heterogeneity in Rubinstein-Taybi syndrome: delineation of the phenotype of the first patients carrying mutations in EP300. J Med Genet 2007; 44: 327-333.

5 Roelfsema JH, Peters DJ: Rubinstein-Taybi syndrome: clinical and molecular overview. Expert Rev Mol Med 2007; 9: 1-16.

6 Stankiewicz P, Beaudet AL: Use of array CGH in the evaluation of dysmorphology, malformations, developmental delay, and idiopathic mental retardation. Curr Opin Genet Dev 2007; 17: 182-192.

7 Shinawi M, Cheung SW: The array CGH and its clinical applications. Drug Discov Today 2008; 13: 760-770

8 Glessner JT, Wang K, Cai G et al: Autism genome-wide copy number variation reveals ubiquitin and neuronal genes. Nature 2009; 459: 569-573.

9 Zhang F, Carvalho CM, Lupski JR: Complex human chromosomal and genomic rearrangements. Trends Genet 2009; 25: 298-307.

10 del Gaudio D, Yang Y, Boggs BA et al: Molecular diagnosis of Duchenne/Becker muscular dystrophy: enhanced detection of dystrophin gene rearrangements by oligonucleotide array-comparative genomic hybridization. Hum Mutat 2008; 29: 1100-1107.

11 Zimmermann N, Acosta AM, Kohlhase J, Bartsch O: Confirmation of EP300 gene mutations as a rare cause of Rubinstein-Taybi syndrome. Eur J Hum Genet 2007; 15 837-842.

12 Foley P, Bunyan D, Stratton J, Dillon M, Lynch SA: Further case of Rubinstein-Taybi syndrome due to a deletion in EP300. Am J Med Genet A 2009; 149A: 997-1000.
13 El-Hattab AW, Smolarek TA, Walker ME et al: Redefined genomic architecture in $15 q 24$ directed by patient deletion/duplication breakpoint mapping. Hum Genet 2009; 126: 589-602.

14 Ou Z, Kang SH, Shaw CA et al: Bacterial artificial chromosome-emulation oligonucleotide arrays for targeted clinical array-comparative genomic hybridization analyses. Genet Med 2008; 10: 278-289.

15 Schouten JP, McElgunn CJ, Waaijer R, Zwijnenburg D, Diepvens F, Pals G: Relative quantification of 40 nucleic acid sequences by multiplex ligation-dependent probe amplification. Nucleic Acids Res 2002; 30: e57.

16 Nowakowska BA, Obersztyn E, Szymańska K et al: Severe mental retardation, seizures, hypotonia, thinning of corpus callosum, and delay of white matter myelination due to deletions of MEF2C. Am J Med Genet B 2010; 153B: 1042-1051.

17 Wisniowiecka-Kowalnik B, Nesteruk M, Peters SU et al: Intragenic rearrangements in NRXN1 in three families with autism spectrum disorder, developmental delay, and speech delay. Am J Med Genet B Neuropsychiatr Genet 2010; 153B: 983-993.

18 Coupry I, Roudaut C, Stef M et al: Molecular analysis of the CBP gene in 60 patients with Rubinstein-Taybi syndrome. J Med Genet 2002; 39: 415-421.

19 Chiang PW, Lee NC, Chien N, Hwu WL, Spector E, Tsai AC: Somatic and germ-line mosaicism in Rubinstein-Taybi syndrome. Am J Med Genet A 2009; 149A: 1463-1467.

20 Marangi G, Leuzzi V, Orteschi D et al: Duplication of the Rubinstein-Taybi region on 16 p13.3 is associated with a distinctive phenotype. Am J Med Genet A 2008; 146A: 2313-2317.

21 Stef M, Simon D, Mardirossian B et al: Spectrum of CREBBP gene dosage anomalies in Rubinstein-Taybi syndrome patients. Eur J Hum Genet 2007; 15: 843-847.

22 Lachmeijer AM, Arngrimsson R, Bastiaans EJ et al: A genome-wide scan for preeclampsia in the Netherlands. Eur J Hum Genet 2001; 9: 758-764. 